\title{
Ação de Justicia pectoralis Jacq. (Acanthaceae) no tratamento de infecções do trato
}

\section{respiratório: uma revisão de literatura}

\author{
Action of Justicia pectoralis Jacq. (Acanthaceae) in the treatment of respiratory tract infections: a \\ literature review
}

Acción de Justicia pectoralis Jacq. (Acanthaceae) en el tratamiento de las infecciones del tracto respiratorio: revisión de la literatura

Recebido: 11/11/2021 | Revisado: 19/11/2021 | Aceito: 23/11/2021 | Publicado: 05/12/2021

Fiamma Acsa de Siqueira Araújo ORCID: https://orcid.org/0000-0003-2907-1964

Universidade Federal de Pernambuco, Brasil E-mail: fiamma.acsa@ufpe.br

Rodhes Geovana de Almeida Rocha ORCID: https://orcid.org/0000-0003-3332-7889 Universidade Federal de Pernambuco, Brasil E-mail: rodhes.rocha@ufpe.br

Luis André de Almeida Campos ORCID: https://orcid.org/0000-0002-9849-922X Universidade Federal de Pernambuco, Brasil E-mail: luis.andre@ufpe.br

Danielle Feijó de Moura ORCID: https://orcid.org/0000-0002-8258-0541 Universidade Federal de Pernambuco, Brasil E-mail: danielle.feijo@hotmail.com

Marcela Araújo Pereira

ORCID: https://orcid.org/0000-0002-6584-3872 Universidade Federal de Pernambuco, Brasil E-mail: apereira.marcela@gmail.com René Duarte Martins

ORCID: https://orcid.org/0000-0002-9444-3501

Universidade Federal de Pernambuco, Brasil E-mail: rene.duarte@ufpe.br

Isabella Macário Ferro Cavalcanti

ORCID: https://orcid.org/0000-0002-7889-3502

Universidade Federal de Pernambuco, Brasil E-mail: isabella.cavalcanti@ufpe.br

\begin{abstract}
Resumo
Há milhares de anos a humanidade a fim de garantir a sua sobrevivência, utilizava as plantas em seu cotidiano para auxiliar na cura e no tratamento de diversas patologias. Essa prática médica integrativa é conhecida como fitoterapia e vem crescendo ao longo dos anos como uma alternativa complementar bastante promissora. Diversos extratos apresentam um amplo espectro de ação profilática frente a várias infecções, assim Justicia pectoralis Jacq. (Acanthaceae) é uma erva medicinal encontrada em diversos países da América, conhecida no Brasil popularmente como "chambá", "anador" ou "trevo-cumaru", sendo bastante empregada no tratamento de distúrbios respiratórios como tosse, asma e bronquite. Assim, o objetivo desse estudo foi realizar uma revisão da literatura sobre a ação de Justicia pectoralis no tratamento de infecções do trato respiratório. Inicialmente, foi realizada uma busca por artigos indexados nas bases de dados Lilacs, PubMed, Google Acadêmico, ScienceDirect, Scielo e Medline publicados entre os anos de 2010 a 2020 nos idiomas português, inglês e espanhol. O extrato de Justicia pectoralis apresenta atividade frente a várias bactérias, dentre elas, algumas que provocam infecções do trato respiratório como, por exemplo, Acinetobacter baumannii, Klebsiella pneumoniae e Staphylococcus aureus. A 1,2 benzopirona e a 7-hidroxicumarina são os fitocompostos majoritários dessa planta responsáveis pelas ações anti-inflamatórias, broncodiladoras, antihistamínicas e analgésicas que contribuem no alívio dos sintomas das infecções respiratórias. Por fim, uma vez que Justicia pectoralis Jacq. (Acanthaceae) já possui ampla utilização na medicina popular e importantes destas atividades se correlacionam com os achados in vitro, faz-se necessário o aprofundamento de estudos que direcionem para o futuro uso clínico desta espécie.
\end{abstract}

Palavras-chave: Fitoterapia; Chambá; Asma; Doenças respiratórias. 


\begin{abstract}
For thousands of years, humanity, in order to ensure its survival, used plants in their daily lives to help cure and treat various pathologies. This integrative medical practice is known as herbal medicine and has grown over the years as a very promising complementary alternative. Several extracts have a broad spectrum of prophylactic action against various infections, such as Justicia pectoralis Jacq. (Acanthaceae) is a medicinal herb found in several countries in America, popularly known in Brazil as "chamba", "anador" or "trevo-cumaru", being widely used in the treatment of respiratory disorders such as cough, asthma, and bronchitis. Thus, the aim of this study was to conduct a literature review on the action of Justicia pectoralis in the treatment of respiratory tract infections. Initially, a search was performed for articles indexed in Lilacs, PubMed, Google Scholar, ScienceDirect, Scielo and Medline databases published between 2010 and 2020 in Portuguese, English and Spanish. Justicia pectoralis extract is active against several bacteria, including some that cause respiratory tract infections, such as Acinetobacter baumannii, Klebsiella pneumoniae and Staphylococcus aureus. 1,2 benzopyrone and 7-hydroxycoumarin are the major phytocompounds of this plant responsible for the anti-inflammatory, bronchodilator, antihistamine, and analgesic actions that contribute to the relief of symptoms of respiratory infections. Finally, since Justicia pectoralis Jacq. (Acanthaceae) is already widely used in folk medicine and important of these activities are correlated with in vitro findings, it is necessary to deepen studies that direct the future clinical use of this species.
\end{abstract}

Keywords: Phytotherapy; Chambá; Asthma; Respiratory diseases.

\title{
Resumen
}

Durante miles de años, la humanidad, con el fin de asegurar su supervivencia, utilizó plantas en su vida diaria para ayudar a curar y tratar diversas patologías. Esta práctica médica integradora se conoce como medicina herbal y ha crecido a lo largo de los años como una alternativa complementaria muy prometedora. Varios extractos tienen un amplio espectro de acción profiláctica frente a diversas infecciones, como Justicia pectoralis Jacq. (Acanthaceae) es una hierba medicinal presente en varios países de América, conocida popularmente en Brasil como "chamba", "anador" o "trevo-cumaru", siendo ampliamente utilizada en el tratamiento de trastornos respiratorios como tos, asma y bronquitis. Así, el objetivo de este estudio fue realizar una revisión de la literatura sobre la acción de Justicia pectoralis en el tratamiento de las infecciones del tracto respiratorio. Inicialmente, se realizó una búsqueda de artículos indexados en las bases de datos Lilacs, PubMed, Google Scholar, ScienceDirect, Scielo y Medline publicados entre 2010 y 2020 en portugués, inglés y español. El extracto de Justicia pectoralis es activo contra varias bacterias, incluidas algunas que causan infecciones del tracto respiratorio, como Acinetobacter baumannii, Klebsiella pneumoniae y Staphylococcus aureus. La 1,2 benzopirona y la 7-hidroxicumarina son los principales fitocompuestos de esta planta responsables de las acciones antiinflamatorias, broncodilatadoras, antihistamínicas y analgésicas que contribuyen al alivio de los síntomas de las infecciones respiratorias. Finalmente, desde Justicia pectoralis Jacq. (Acanthaceae) ya tiene un amplio uso en la medicina popular y importantes de estas actividades se correlacionan con los hallazgos in vitro, es necesario profundizar los estudios que orienten el uso clínico futuro de esta especie.

Palabras clave: Fitoterapia; Chambá; Asma; Enfermedades respiratorias.

\section{Introdução}

A utilização de plantas na medicina faz parte de uma cultura histórica e popular, construída há séculos a partir da sabedoria do senso comum, que articula saúde e cultura as quais encontram-se enraizadas até os dias atuais. Historicamente inserida na vida do homem desde a pré-história, o uso de espécies medicinais como medicamento é considerado como uma das práticas terapêuticas mais antigas e rentáveis que têm acompanhado a humanidade ao longo dos anos (Melo et al., 2017; Sarmento Gadelhara, Borges Maracajá, \& Martins, 2015)

Caracterizada pela utilização de plantas em diversas formas farmacêuticas, a fitoterapia levou a descobertas promissoras da ação terapêutica para o ser humano. As plantas medicinais são constituídas por substâncias bioativas, denominados de metabólitos secundários que podem ser responsáveis por ações profiláticas para algumas patologias (Mesquita, Pinto, \& Moreira, 2017). Diante disso, o interesse por essa prática vem aumentando entre pesquisadores, serviços de saúde e usuários na possibilidade de garantir uma terapia eficiente com menos efeitos colaterais (Costa et al., 2019; De Lima \& Macedo, 2019)

Diversas patologias têm sido tratadas através da utilização de plantas, dentre elas vale destacar as que estão relacionadas ao trato respiratório. Doenças relacionadas a esse sistema são caracterizadas pela obstrução da passagem do ar e por isso, representam um dos maiores problemas de saúde mundial (Lumertz, Rossato, Birollo, \& Soratto, 2017; Pabón, Rodríguez, \& Hernández-Rodríguez, 2017; Prato, Silveira, Neves, \& Buboltz, 2014). 
No Brasil, as infecções associadas ao aparelho respiratório juntamente com as doenças cardiovasculares ocupam a segunda e terceira principal causa de internações hospitalares, respectivamente e podem estar presentes em todas as faixas etárias, com casos graves se constituindo como importantes causas de adoecimentos, internações e óbitos (Brambilla, Endo, Cortez, \& Filho, 2017; Dias, Mendonça, Pinto, Borges, \& Oliveira, 2020; Mesquita et al., 2017).

Na perspectiva de um tratamento natural e com boa aceitação popular, uma espécie de planta medicinal que possui grande expressividade é Justicia pectoralis Jacq. (Acanthaceae), uma planta utilizada em vários países devido ao seu potencial terapêutico frente a algumas infecções (Leal, Silva, \& Viana, 2017; Moura et al., 2017). Essa planta é uma erva medicinal conhecida no Brasil popularmente como "chambá", "anador" ou "trevo-cumaru" pertencente à família Acanthaceae, está distribuída em regiões tropicais da América Latina podendo atingir 15 a $30 \mathrm{~cm}$ de altura. Justicia pectoralis Jacq. (Acanthaceae) é caracterizada morfologicamente pela presença de caules reptantes de coloração esverdeada, folhas opostas, flores com inflorescência terminais e frutos que apresentam facilidade de se multiplicar por estacas ou ramos proporcionando uma fácil propagação da espécie (Aoyama \& Indriunas, 2015; Marcos, Bezerra, \& Tertuliano, 2006; Venâncio et al., 2011; Venegas-Casanova et al., 2018).

No Nordeste do Brasil, o xarope de chambá é bastante utilizado no tratamento de asma, tosse e bronquite e por isso, é uma planta útil para o tratamento de doenças respiratórias. Esse xarope é largamente produzido e distribuído pelo programa público de fitoterapia denominado Farmácias Vivas, visando o cultivo e distribuição de plantas medicinais, com possibilidade de desenvolvimento de medicamentos fitoterápicos para o uso do Sistema Único de Saúde (Leal et al., 2017; Moura et al., 2017).

Desse modo, o objetivo do presente estudo foi realizar uma revisão da literatura sistemática sobre a ação de Justicia pectoralis Jacq. (Acanthaceae) no tratamento de infecções do trato respiratório.

\section{Metodologia}

Trata-se de uma revisão de literatura sistemática com base nas seguintes etapas: identificação do tema e desenvolvimento da questão norteadora; estabelecimento de critérios de inclusão e exclusão, análise e seleção de estudos; interpretação de dados e resultados; apresentação da revisão (Figura 1). A pergunta norteadora foi: "Qual é a ação de Justicia pectoralis Jacq. (Acanthaceae) no tratamento de infecções relacionadas ao trato respiratório?”. Incialmente foi realizada a busca por artigos indexados em bibliotecas virtuais internacionais, Literatura Latino-Americana e caribenha em Ciências da Saúde (Lilacs), Biblioteca Nacional de Medicina dos EUA (PubMed), Google Acadêmico, ScienceDirect, Scientific Electronic Library Online (Scielo) e Medline.

Os critérios de inclusão adotados foram artigos completos publicados em inglês, português e espanhol relacionados ao tema proposto e indexados nessas bases de dados, publicados entre 2010 e 2020. Por sua vez, foram utilizados estudos repetidos como critérios de exclusão, que não abordam o tema proposto, incompleto, duplicatas, monografias, publicações de eventos e teses. Foram utilizados os seguintes descritores: doenças do trato respiratório, fitoterapia, Justicia pectoralis e atividade antibacteriana. 
Figura 1: Fluxograma da aplicação dos critérios de inclusão e exclusão de artigos utilizados na revisão de literatura deste estudo. Modificado de Pitta e colaboradores, 2021 (Pitta et al., 2021).

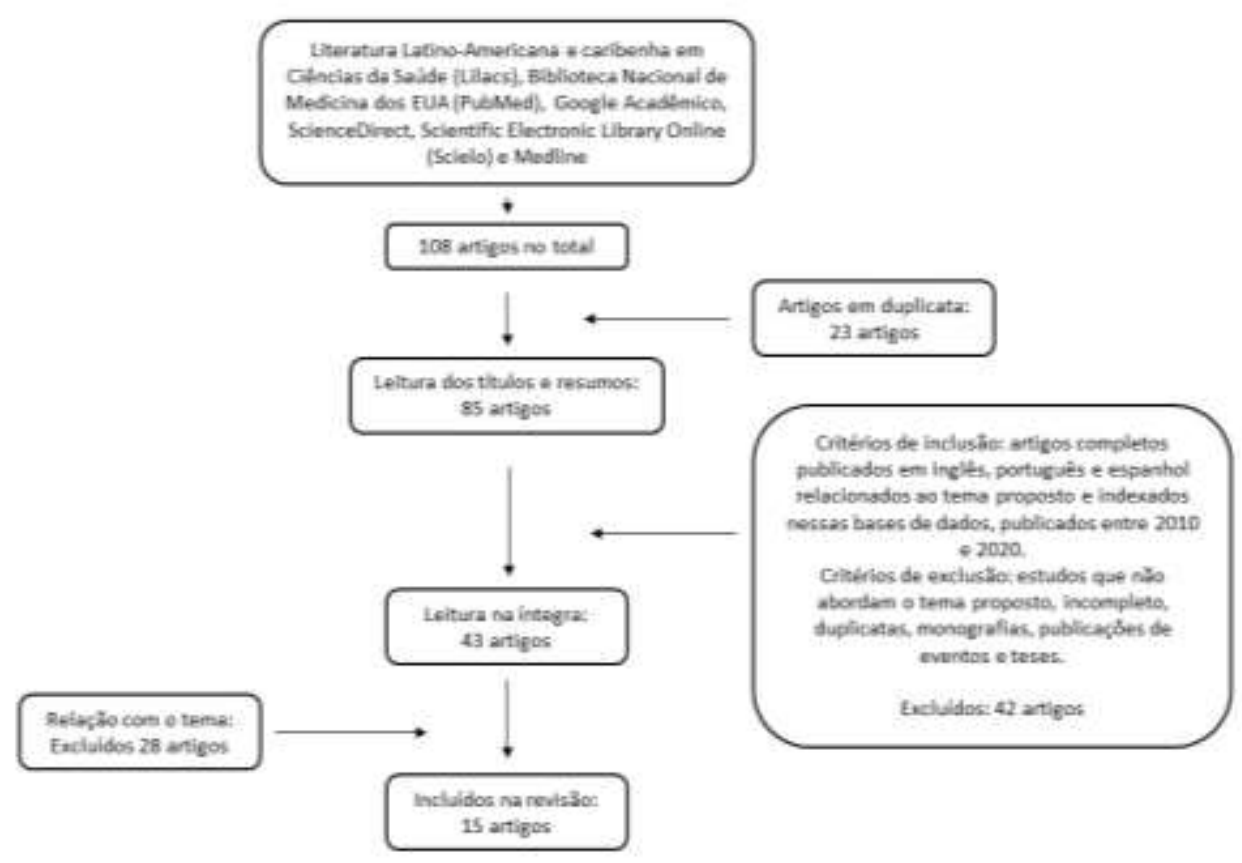

Fonte: Autores.

\section{Resultados e Discussão}

\section{O uso de plantas medicinais no tratamento de infecções do trato respiratório}

Uma das maiores preocupações globais existentes referente à saúde humana são as doenças infecciosas, pois elas são consideradas como uma das patologias mais comuns e mais nocivas ao homem. Atualmente, as infecções são apontadas como um grande desafio à saúde pública, se destacando entre as causas mais frequentes de internações e óbitos nos sistemas de saúde (Becker, Hu, \& Biller-Andorno, 2006; G.J., B.R., \& CASE, 2016; Hespanhol, Ramos, Junior, de Araújo, \& Martins, 2019; Junqueira \& Duarte, 2012; Da Silva et al., 2020).

De acordo com a Organização Mundial da Saúde (OMS), as infecções do trato respiratório são aquelas que afetam a passagem do ar através das vias respiratórias, incluindo as nasais, brônquios e pulmões. Dentre as infecções bacterianas, virais ou fúngicas que acometem a saúde da humanidade, destacam-se as que estão relacionadas ao aparelho respiratório (Prudente \& Moura, 2013).

As infecções referentes as vias aéreas estão divididas em duas partes segundo a localização anatômica. As infecções do trato superior (ITRs) compreendem as doenças mais conhecidas pela população, incluindo resfriado, sinusite e amigdalite, e as infecções do trato respiratório inferior (ITRi) que incluem bronquite aguda, bronquiolites e pneumonia. As infecções que estejam relacionadas ao trato superior são as mais frequentes e com menos risco de morte, já as infecções que acometem a porção inferior são as responsáveis por ocasionar quadros mais graves à saúde (De Amorim Corrêa et al., 2017; Filho, Silva, Santos, Dall'acqua, \& Souza, 2017; Grief, 2013).

Considerada uma doença de alto contágio devido a sua alta capacidade de se propagar por meio de partículas contaminadas liberadas no ar, seja por espirros ou bocejos, as infecções respiratórias agudas (IRAs) além de comprometerem estruturas do sistema respiratório, também são as responsáveis por $98 \%$ das causas de morbimortalidade em bebês, crianças, 
adultos e idosos (Malosh, Martin, Ortiz, \& Monto, 2018; Troeger et al., 2018; World Health Organization, 2014; Zumla \& Niederman, 2015).

Dentre os inúmeros tratamentos existentes para combater as infecções, a utilização das plantas medicinais é bastante promissora. Nos últimos anos a fitoterapia vem sendo valorizada nos programas dos sistemas únicos de saúde como uma terapia medicamentosa complementar já que, os extratos de plantas apresentam um amplo espectro de ação terapêutica em diversas patologias, principalmente nas infecções respiratórias (Pietruszewska, Barańska, \& Wielgat, 2018; Ribeiro, 2019).

Nesse contexto, diversas plantas têm sido utilizadas sob uma variedade de sintomas relacionados as IRAs, como febre, tosse, dores de garganta, bronquite, pneumonias e entre outra. Observando a eficácia desses vegetais frente a essas infecções, pesquisadores de todo o mundo se dedicaram a analisar esses extratos vegetais para trazerem informações que podem contribuir futuramente para a descoberta ou desenvolvimento de novos agentes antimicrobianos para o tratamento dessas infecções que comprometem o aparelho respiratório (Lemos et al., 2016).

Amber e colaboradores (2017) realizaram um estudo de revisão da literatura para avaliar a atividade antibacteriana in vitro de plantas etnomedicinais da região do Himalaia frente a patógenos causadores de bronquite. Nesse estudo foram identificadas 6 plantas que possuíam compostos como por exemplo, monoterpenóides, flavonóides, triterpenóides e glicosídeos com forte potencial antiviral (Amber, Adnan, Tariq, \& Mussarat, 2017).

Nas análises realizada por Tseliou e colaboradores (2019) ao utilizarem uma combinação de óleos essenciais à base de plantas aromáticas em azeite extra virgem para o tratamento de sintomas graves em pacientes com ITRs, os autores puderam observar uma atividade antiviral direta contra uma ampla gama de vírus respiratórios in vitro, e conclui que a combinação realizada com três espécies de plantas aromáticas apresenta um grande potencial para ser usado como um fitoterápico contra a gripe, vírus e rinovírus (Tseliou, Pirintsos, Lionis, Castanas, \& Sourvinos, 2019).

Em outro estudo realizado por Prudente e Moura (2013) ao verificar cinco espécies de plantas bastante utilizada em doenças do trato respiratório no sudeste do Brasil, foi possível observar que as substâncias isoladas desses vegetais conseguiram inibir os patógenos bacterianos que são um dos responsáveis por ocasionar essas infecções. Os autores afirmam ainda que esses resultados convergem com as indicações que são apontadas pela população referente ao seu uso popular (Prudente \& Moura, 2013).

Dentre as inúmeras variedades de plantas que são utilizadas como uma terapia medicamentosa frente as infecções do trato respiratório, vale ressaltar a Justicia pectoralis Jacq. (Acanthaceae).

\section{Botânica e distribuição geográfica de Justicia pectoralis Jacq. (Acanthaceae)}

A família Acanthaceae é considerada como uma fonte de princípios ativos para a medicina tradicional e apresenta uma distribuição pantropical compreendendo cerca de 275 gêneros e mais de 4000 espécies que estão amplamente distribuídas nos trópicos em todo o mundo, porém, apresenta poucos representantes nas regiões temperadas. O Brasil apresenta um dos maiores centros da biodiversidade mundial dessa família e se destaca com aproximadamente 40 gêneros e 550 espécies, com ocorrência em todas as formações vegetais, principalmente nos domínios das florestas pluviais (Costa et al., 2019; Liu et al., 2018; McDade, Daniel, \& Kiel, 2008; Profice \& Andreata, 2011).

Justicia pectoralis Jacq. (Acanthaceae) é uma erva medicinal pertencente à família Acanthaceae cultivada em diversos países. Essa espécie tem origem em locais de clima úmido da América Tropical, assim ela passou a ser cultivada em diversas regiões com maiores registros em países da América do Central e do Sul, como por exemplo, México, Jamaica, Cuba, Venezuela, Trindad, Oeste do Equador, Norte e Nordeste do Brasil e em ilhas na região do oeste da Índia (Cameron, Jacob, Thomas, \& Levy, 2015; Oliveira, Xavier, Silva, \& Andrade, 2000; Venâncio et al., 2011). 
Conhecida no Brasil popularmente como "chambá", "anador" ou "trevo-cumaru", Justicia pectoralis Jacq. (Acanthaceae) é uma planta verde, sub-ereta e perene com caules reptantes, ou seja, que crescem emitindo brotos de maneira rastejante junto ao solo. Essa planta é florífera com folhagem ornamental, apresenta de 15 a 30 centímetros de altura possuindo inflorescência terminal, paniculada com flores em formas de cachos e a corola podendo variar a coloração de lilás a violeta (Fonseca, Silva, \& Leal, 2010; Lorenzi \& Matos, 2008; Marcos et al., 2006; Wasshausen \& Wood, 2004).

O caule apresenta uma coloração verde com tricomas esbranquiçados com a presença de nós inferiores com as raízes adventícias, ou seja, que não são originadas a partir da radícula do embrião e sim de outras partes da planta. Esse vegetal apresenta fruto do tipo cápsula deiscente e contém uma facilidade de se multiplicar por estacas ou ramos enraizados crescendo em local formando aglomerados globóides que podem chegar até 40 centímetros de altura (Leal et al., 2017; Lorenzi \& Matos, 2008; Oliveira et al., 2000).

Em sua filotaxia essa planta apresenta folhas opostas de coloração verde, apresentando na sua superfície externa com nervura central sulcada na face adaxial ou superior e verde clara na superfície abaxial ou inferior. Em estudos da lâmina foliar realizados com microscopia eletrônica de varredura observou-se tricomas tectores com formatos arredondados e tricomas tectores subsésseis de quatro células abaxiais (Aoyama \& Indriunas, 2015).

\section{Constituintes de Justicia pectoralis Jacq. (Acanthaceae)}

Consideradas como laboratórios naturais, as plantas apresentam inúmeras quantidades de constituintes químicos com diversas aplicações biológicas. Essas substâncias bioativas derivadas do metabolismo secundário encontradas nesses vegetais com diversas propriedades profiláticas são utilizadas nas indústrias farmacêuticas como alternativas medicamentosas promissoras na atenção primária à saúde (Bertarello Zeni, Ana Lúcia; Mattos, Gerson; Santa Helena, Ernani Tiaraju; Parisotto, 2017; Pérez Alonso \& Jiménez, 2011; Yordan Martínez Aguilar, Rodríguez, Manuel Almeida Saavedra, Espinosa, \& Yero, 2012).

O gênero Justicia contém uma grande diversidade de compostos químicos com propriedades terapêuticas. Triagens fitoquímicas realizadas nas partes aéreas desse vegetal revelaram a presença de açúcares redutores, flavonóides, saponinas, carotenos, triterpenos, esteróides e cumarinas. Acredita-se que as cumarinas são os princípios ativos que atuam nos benefícios profiláticos dessa espécie na medicina tradicional (Araújo, Faria, \& Safadi, 2014; J. Rodríguez Chanfrau, López Hernández, \& Gil Apan, 2008).

Justicia pectoralis Jacq. (Acanthaceae) apresenta vários componentes cumarínicos descritos na literatura como a 1,2benzopirona, di-hidrocumarina,7-hidroxicumarina, 2-metoxi-4-cromono, 3-isocromanona, ácido 3-fenilpropiônico, ácido propiônico 3-(2-hidroxifenil) e ácido propiônico metil éster 3-(2-hidroxifenil), obtidos das partes aéreas da planta através de cromatografia gasosa de massa (Leal et al., 2017; MacRae \& Towers, 1984; de Vries, Tauscher, \& Wurzel, 1988).

Dentre esses constituintes presentes nessa planta, vale destacar a 1,2-benzopirona e a 7-hidroxicumarina que são os principais responsáveis pelas atividades terapêuticas de Justicia pectoralis. Essas substâncias já foram descritas com ações anti-inflamatória, antimicrobiana, antitumoral, antioxidante, antiviral, vasodilatadora, anti-histamínico, hipoglicemiante, broncodilatadora, expectorante e sedativa (Cameron et al., 2015; Corrêa \& Alcântara, 2012; Furtado, Amorim, Fernandes, \& Oliveira, 2015; Guimarães, Silva, Lima, Magalhães, \& Figueiredo, 2020).

A cumarina 1,2- benzopirona, considerada a molécula mãe das cumarinas, é um composto encontrado em diversas variedades de microrganismos, plantas e animais. A 7-hidroxicumarina classificada como um dos principais produtos biotransformadores da 1,2 benzopirona, também é conhecida como umbeliferona, um composto orgânico natural pertencente à classe das cumarinas que também é encontrado em vários vegetais. Quimicamente essas moléculas são compostos fenólicos formados pela fusão de um benzeno e de um anel $\alpha$-pirona. Derivados do metabolismo secundário da planta, esses 
fitocompostos apresentam inúmeras atividades farmacológicas (Jia et al., 2019; J. E. Rodríguez Chanfrau \& Rodríguez Ferrada, 2014; Wu et al., 2020).

\section{O uso de Justicia pectoralis Jacq. (Acanthaceae) na medicina}

Justicia pectoralis é uma erva medicinal com uma longa história de uso tradicional em vários países da América Central e Sul bastante utilizada para diferentes fins terapêuticos. Esse vegetal vem sendo empregado como um fitofármaco tornando-se uma alternativa medicamentosa mais saudável e com menos efeitos colaterais em diversas enfermidades na América (Moura et al., 2017; Rockenbach et al., 2018).

$\mathrm{Na}$ Costa Rica, os extratos secos de Justicia pectoralis conhecidos como tilo são utilizados no alívio de sintomas da menopausa ou em outras patologias que estão relacionadas a saúde da mulher. No Panamá, essa planta é empregada na medicina tradicional em forma de chá pelas populações para alívios de dores de cabeça, estômago e pernas (Benítez-Benítez et al., 2020; Leal et al., 2017; Locklear et al., 2010).

Além disso, no Caribe, as folhas da Justicia pectoralis são comercializadas como ansiolíticos em situações de ansiedade, estresse e dificuldade para dormir. Na medicina tradicional Jamaicana, essa planta é utilizada em forma de chá para reduzir a dificuldade da respiração em pacientes asmáticos. Tradicionalmente, em Trinidad e Tobago, são usadas em homens no tratamento de doenças relacionadas a próstata como também é empregada como expectorante no tratamento de resfriados (Cameron et al., 2015; Govín, Hernandez, Figueredo, \& Ferradá, 2003; Venegas-Casanova et al., 2018).

Em Cuba, Justicia pectoralis faz parte do arsenal botânico medicinal autorizado pelo Ministério Público do país, sendo utilizada principalmente como sedativo atuando como um medicamento capaz de reduzir a ansiedade, exercendo também um efeito calmante e expectorante. Devido as suas propriedades terapêuticas, em 1991, essa espécie passou a ser processada pelas indústrias farmacêuticas cubanas contribuindo como alternativas profiláticas na assistência à saúde. Na América do sul, essa planta é considerada, na Venezuela, como adstringente, anti-hemorrágica, expectorante e calmante, suas folhas são usadas na cicatrização de feridas como também é empregada no tratamento de doenças de fígado(Ball et al., 2000; Balseca Mata, 2017).

No Equador, seus extratos são utilizados para o tratamento de dores relacionadas ao ciclo menstrual, tosses e resfriados e, na Colômbia, as partes aéreas desse vegetal também são comumente usados no tratamento de diabetes, doenças relacionadas à próstata, como também em infecções (Corrêa \& Alcântara, 2012; Locklear et al., 2010; Venegas-Casanova et al., 2018).

No Brasil, mais especificamente no Nordeste do país, essa planta é conhecida como "chambá", "trevo-cumaru" e "anador". O xarope dessa planta é popularmente utilizado no tratamento de asma, tosse e bronquite. Esse xarope é largamente produzido e compartilhado pelo programa público de fitoterapia denominado Farmácias Viva, visando o desenvolvimento de medicamentos fitoterápicos e, para o uso do Sistema Único de Saúde (Leal et al., 2017; Moura et al., 2017; Rosina Maria Carvalho Caminha Muniz, Andre Luiz Cunha Cavalcante, Luis Mario Silva Pereira, Francisca Clea Florenco de Sousa, Silvania Maria Mendes de Vasconcellos, Danielle Silveira Macedo, Glauce Socorro de Barros Viana, 2012).

\section{O uso de Justicia pectoralis Jacq. (Acanthaceae) nas infeç̧ões relacionadas ao trato respiratório}

As infecções do trato respiratório continuam sendo um grande desafio à saúde pública constituindo uma das maiores causas de mortalidade em todo o mundo. Devido a gama de agentes etiológicos existentes e as dificuldades que são encontradas para se estabelecer um diagnóstico preciso, na maioria das vezes, o tratamento destas infecções são iniciados empiricamente pelas populações, recorrendo-se as plantas medicinais (Semenya \& Maroyi, 2018). 
$\mathrm{O}$ uso desses vegetais na medicina tradicional é considerado como um dos recursos terapêuticos mais antigos utilizados pelo homem nos cuidados primários à saúde em diversas patologias. Estudos descritos na literatura afirmam que Justicia pectoralis é empregada tradicionalmente pelas populações no tratamento das infecções do trato respiratório como a tosse, asma e bronquite (Andrade, Casali, \& Cecon, 2012; Benitez, Silva, \& Alvares, 2016; Fonseca et al., 2010; Leal et al., 2017).

Levantamentos realizados por Ferreira e colaboradores (2015) ao analisarem espécies vegetais utilizadas para fins medicinais por estudantes de diferentes realidades de ensino médio incluindo a comunidade quilombola, constataram que dentre as ervas citadas, a Justicia pectoralis foi mencionada por esses povos no preparo de chás em casos de bronquites, dores, gripe, aftas e azias (Ferreira, Batista, \& Pasa, 2015).

Um outro estudo realizado por Ruzza e colaboradores (2014) relatou que moradores do município de Alta Floresta localizado no estado do Mato Grosso do Sul utilizam o chá das folhas de Justicia pectoralis para o tratamento da gripe (Ruzza, Göttert, Rossi, Dardengo, \& Silva, 2014).

Sousa e Silva (2015), ao buscarem conhecer as principais plantas medicinais utilizadas por mulheres idosas no município de Quixeré no estado do Ceará, verificaram que Justicia pectoralis faz parte desse arsenal terapêutico utilizada por essas idosas em forma de lambedor no tratamento de pneumonia (Saraiva et al., 2015).

Várias populações fazem o uso popular da Justicia pectoralis Jacq. (Acanthaceae) no tratamento de infecções do trato respiratório. Estudos afirmam que as cumarinas, substância presente nessa planta, apresentam ações farmacológicas antivirais, porém no presente momento ainda não há estudos científicos que relatem a ação direta dessas substâncias bioativas frente ao vírus influenza, vírus que provoca a gripe. Já foi relatado que a 7-hidroxicumaria e/ou umbeliferona, outras moléculas também encontradas nessa planta, induziram a produção de citocinas com atividade modulatória em camundongos o que resultou num alívio da infecção (Filho et al., 2017; Guimarães et al., 2020; Kurokawa, Watanabe, Shimizu, Sawamura, \& Shiraki, 2010) (Kurokawa et al., 2010; Filho et al., 2017; Guimarães et al., 2020).

A pneumonia, também foi uma das infecções citadas pelas populações que faz o uso dessa espécie em seu tratamento. Uma análise científica retratada na literatura afirma que o extrato orgânico da Justicia pectoralis apresentou atividade bacteriostática numa concentração de $500 \mu \mathrm{g} / \mathrm{mL}$ frente as cepas de Acinetobacter baumannii e Klebsiella pneumoniae. Em relação a outros microrganismos que também são responsáveis por ocasionar essa infecção como Streptococcus pneumoniae e Haemophilus influenza, ainda não foram cientificamente descritos na literatura a atividade antimicrobiana dos extratos da Justicia pectoralis (Furtado et al., 2015; Rockenbach et al., 2018).

Recentemente, estudos realizados por Guimarães e colaboradores (2020) avaliaram a atividade antimicrobiana do extrato aquoso de Justicia pectoralis em microcápsulas e observaram que na concentração de 5 a $200 \mathrm{mg} / \mathrm{mL}$ houve atividade frente às cepas de Staphylococcus aureus, Listeria monocytogenes, Salmonella enteritidis e Escherichia coli (Guimarães et al., 2020).

Adicionalmente, Justicia pectoralis Jacq. (Acanthaceae) apresenta outros potenciais farmacológicos, com ações antiinflamatórias, analgésicas e broncodiladoras, anti-histamínicas e, a ação conjunta das principais substâncias dessa espécie, justifica o uso desse vegetal no alívio dos sintomas decorrentes de infecções do trato respiratório, como tosse, dores de cabeça, febre, bronquite e asma (Cameron et al., 2015; Mpiana \& Kitadi, 2019; J. E. Rodríguez Chanfrau, López Hernández, Núñez Figueredo, Rodríguez Ferrada, \& Nogueira Mendoza, 2013).

\section{Conclusão}

Devido ao seu potencial farmacêutico com menos efeitos colaterais e por serem consideradas como opção terapêutica de baixo custo, as plantas medicinais vêm crescendo ao longo dos anos auxiliando na cura e no tratamento de diversas 
patologias. Nesse contexto, Justicia pectoralis Jacq. (Acanthaceae) é bastante utilizada, popularmente, no tratamento das infecções relacionadas ao trato respiratório. Componentes cumarínicos majoritários 1,2 benzopirona e a 7-hidroxicumarina presentes nessa espécie dispõem de um arsenal de ações farmacológicos com propriedades terapêuticas com ações antiinflamatórias, broncodiladoras, anti-histamínica e analgésicas o que justifica o uso empírico desse vegetal como um recurso profilático frente a essas infecções.

Por fim, concluímos que há a necessidade de realização de novos estudos científicos mais completos e robustos, que busquem comprovar a atividade antimicrobiana, in vitro e in vivo, dessa planta medicinal com ação direta frente aos mais frequentes patógenos responsáveis por ocasionar essas infecções respiratórias.

\section{Agradecimentos}

F.A.S.A. agradece à Fundação de Apoio à Ciência e Tecnologia do Estado de Pernambuco -FACEPE pela bolsa de iniciação científica. R.G.A.R.R. agradece ao Conselho Nacional de Desenvolvimento Científico e Tecnológico -CNPq. I.M.F.C. agradece ao CPNq (426065/2018-2) por parte do apoio financeiro deste trabalho.

\section{Referências}

Amber, R., Adnan, M., Tariq, A., \& Mussarat, S. (2017). A review on antiviral activity of the Himalayan medicinal plants traditionally used to treat bronchitis and related symptoms. The Journal of pharmacy and pharmacology, 69(2), 109-122.

De Amorim Corrêa, R., De São José, B. P., Malta, D. C., De Azeredo Passos, V. M., França, E. B., Teixeira, R. A., \& Camargos, P. A. M. (2017). Carga de doença por infecções do trato respiratório inferior no Brasil, 1990 a 2015: Estimativas do estudo Global Burden of Disease 2015. Revista Brasileira de Epidemiologia, 20(25000192049), 171-181.

Andrade, F. M. C., Casali, V. W. D., \& Cecon, P. R. C. (2012). Crescimento e produção de cumarina em plantas de chambá (justicia pectoralis jacq.) tratadas com isoterápico. Revista Brasileira de Plantas Medicinais, 14(SPL.ISS), 154-158.

Aoyama, E. M., \& Indriunas, A. (2015). Micromorfologia e anatomia foliar de duas espécies de Justicia L. (Acanthaceae) de uso medicinal Micromorphology and leaf anatomy of two species of Justicia L. (Acanthaceae) that have medicinal use. Revista de Biologia Neotropical, 11 (2), 97.

Araújo, L. L. N., Faria, M. J. M. de, \& Safadi, G. M. V. V. (2014). Prospecção Fitoquímica da espécie justicia pectoralis jacq. var. Stenophylla leonard pertencente à família Acanthaceae. Revista Eletrônica de Ciências Humanas e Tecnologia, 3(2), 4-14.

Ball, T. M., Castro-Rodriguez, J. A., Griffith, K. A., Holberg, C. J., Martinez, F. D., \& Wright, A. L. (2000). Siblings, day-care attendance, and the risk of asthma and wheezing during childhood. The New England journal of medicine, 343(8), 538-543. United States.

Balseca Mata, R. D. (2017). Screening fitoquímico y evaluación de la actividad antimicrobiana de: Catharanthus Roseus (1.) G. Don, Justicia Pectoralis Jacq. y Scoparia Dulcis 1. FIGEMPA: Investigación y Desarrollo, 1(1 SE-Artículos), 68-78. https://revistadigital.uce.edu.ec/index.php/RevFIG/article/view/58

Becker, K., Hu, Y., \& Biller-Andorno, N. (2006). Infectious diseases - a global challenge. International journal of medical microbiology : IJMM, 296(4-5), $179-185$.

Benítez-Benítez, R., Sarria-Villa, R. A., Gallo-Corredor, J. A., Pérez Pacheco, N. O., Álvarez Sandoval, J. H., \& Giraldo Aristizabal, C. I. (2020). Obtención y rendimiento del extracto etanólico de dos plantas medicinales. Revista Facultad de Ciencias Básicas, 15(1), 31-40.

Benitez, L. B., Silva, C. de M. da, \& Alvares, L. da C. (2016). Utilização da hortelã-pimenta como agente no controle de infecções relacionadas à assistência à saúde (IRAS). Scientia Plena, 12(12), 1-9.

Bertarello Zeni, Ana Lúcia; Mattos, Gerson; Santa Helena, Ernani Tiaraju; Parisotto, A. V. (2017). Utilização de plantas medicinais como remédio caseiro na Atenção Primária em Blumenau - SC. Ciência e Saúde Coletiva, 22(8), 2703-2712.

Brambilla, L. Z. S., Endo, E. H., Cortez, D. A. G., \& Filho, B. P. D. (2017). Anti-biofilm activity against staphylococcus aureus mrsa and mssa of neolignans and extract of piper regnellii. Brazilian Journal of Pharmacognosy, 27(1), 112-117. Sociedade Brasileira de Farmacognosia. http://dx.doi.org/10.1016/j.bjp.2016.08.008

Cameron, C., Jacob, A. S., Thomas, E. A., \& Levy, A. S. (2015). Preliminary Investigations of the Anti-asthmatic Properties of the Aqueous Extract of Justicia pectoralis (Fresh Cut). The West Indian medical journal, 64(4), 320-324.

Corrêa, G. M., \& Alcântara, A. F. D. C. (2012). Chemical constituents and biological activities of species of Justicia - a review. Brazilian Journal of Pharmacognosy, 22(May 2011), 220-238.

Costa, N. C., Barbosa Junior, G. C., Morais, P. H. de P. R., Oliveira, É. G., Borges, E. M. A., Gomes, G. C., Da Mata, H. C., et al. (2019). Fitoterápicos na atenção primária à saúde: desafios e perspectivas na atuação médica no SUS. Revista Fitos, 13(2), 117-121. http://revistafitos.far.fiocruz.br/index.php/revistafitos/article/view/770 
Dias, F. L. T., Mendonça, F. D., Pinto, G. M., Borges, I. S. C., \& Oliveira, S. V. de. (2020). Doenças respiratórias no Triângulo Mineiro: Análise epidemiológica e projetiva com a pandemia de COVID-19. Journal of Health \& Biological Sciences, 8(1), 1.

Ferreira, A. L. D. S., Batista, C. A. dos S., \& Pasa, M. C. (2015). Levantamento Etnobotânico nas Diferentes Realidades de Ensino. Biodiversidade, 14(3), 6073 .

Filho, E. B. da S., Silva, A. L., Santos, A. O., Dall'acqua, D. S. V., \& Souza, L. F. B. (2017). Infecções Respiratórias de Importância Clínica: uma Revisão Sistemática. Revista Fimca, 4(1), 7-16.

Fonseca, F. N., Silva, A. H., \& Leal, L. K. A. M. (2010). Justicia pectoralis Jacq., Acanthaceae: Preparation and characterisation of the plant drug including chromatographic analysis by HPLC-PDA. Revista Brasileira de Farmacognosia, 20(6), 871-877.

Furtado, J. M., Amorim, Á. da S., Fernandes, M. V. de M., \& Oliveira, M. A. S. (2015). Atividade Antimicrobiana do Extrato Aquoso de Eucalyptus globulus, Justicia pectoralis e Cymbopogon citratus Frente a Bactérias de Interesse. Journal of Health Sciences, 17(4), 233-237.

Tortora, G. J., Funke, B. R. \& Case, C. L. (2016). Microbiologia - $12^{a}$ Ed. 2016 (12th ed.). Artmed.

Govín, E., Hernandez, L., Figueredo, D., \& Ferradá, C. (2003). Estudio farmacognóstico de Justicia pectoralis Jacq. var. stenophylla Leonard. Revista Cubana de Plantas Medicinales, 8.

Grief, S. N. (2013). Upper respiratory infections. Primary care, 40(3), 757-770. Elsevier Inc. Retrieved from https://pubmed.ncbi.nlm.nih.gov/23958368

Guimarães, T. L. F., Silva, L. M. R. da, Lima, C. de B., Magalhães, F. E. A., \& Figueiredo, E. altina T. de. (2020). Antimicrobial activity of microcapsules with aqueous extract of chambá (Justicia pectoralis Jacq). Revista Ciência Agronômica, 51(2), 1-8.

Hespanhol, L. A. B., Ramos, S. C. de S., Junior, O. C. R., de Araújo, T. S., \& Martins, A. B. (2019). Infection related to health care in an adult intensive care unit. Enfermeria Global, 18(1), 215-254.

Jia, C., Zhang, J., Yu, L., Wang, C., Yang, Y., Rong, X., Xu, K., et al. (2019). Antifungal Activity of Coumarin Against Candida albicans Is Related to Apoptosis. Frontiers in Cellular and Infection Microbiology, 8, 445. Retrieved from https://www.frontiersin.org/article/10.3389/fcimb.2018.00445

Junqueira, R. M. P., \& Duarte, E. C. (2012). Hospitalizations due to ambulatory care-sensitive conditions in the Federal District, Brazil, 2008. Revista de Saúde Pública, 46(5), 761-768.

Kurokawa, M., Watanabe, W., Shimizu, T., Sawamura, R., \& Shiraki, K. (2010). Modulation of cytokine production by 7-hydroxycoumarin in vitro and its efficacy against influenza infection in mice. Antiviral research, 85(2), 373-380. Netherlands.

Leal, L. K. A. M., Silva, A. H., \& Viana, G. S. de B. (2017). Justicia pectoralis, a coumarin medicinal plant have potential for the development of antiasthmatic drugs? Revista Brasileira de Farmacognosia, 27(6), 794-802. Sociedade Brasileira de Farmacognosia. http://dx.doi.org/10.1016/j.bjp.2017.09.005

Lemos, I. C. S., Delmondes, G. de A., Dos Santos, A. D. F., Santos, E. S., de Oliveira, D. R., de Figueiredo, P. R. L., Alves, D. de A., et al. (2016). Ethnobiological survey of plants and animals used for the treatment of acute respiratory infections in children of a traditional community in the municipality of Barbalha, Ceará, Brazil. African journal of traditional, complementary, and alternative medicines : AJTCAM, 13(4), 166-175.

De Lima, W., \& Macedo ${ }^{1}$, R. (2019). Uso da fitoterapia no tratamento de doenças crônicas não transmissíveis: revisão integrativa. ReBIS [Internet], 1(3), 3643.

Liu, B., Yang, Y., Liu, H., Xie, Z., Li, Q., Deng, M., Li, F., et al. (2018). Screening for cytotoxic chemical constituents from Justicia procumbens by HPLCDAD-ESI-MS and NMR. Chemistry Central journal, 12(1), 6.

Locklear, T. D., Huang, Y., Frasor, J., Doyle, B. J., Perez, A., Gomez-Laurito, J., \& Mahady, G. B. (2010). Estrogenic and progestagenic effects of extracts of Justicia pectoralis Jacq., an herbal medicine from Costa Rica used for the treatment of menopause and PMS. Maturitas, 66(3), 315-322.

Lorenzi, H., \& Matos, F. J. de A. (2008). Plantas medicinais no Brasil : nativas e exóticas. Nova Odessa: Instituto Plantarum.

Lumertz, D., Rossato, A. L., Birollo, I. V. B., \& Soratto, M. T. (2017). A utilização de plantas medicinais por pacientes com doenças respiratórias crônicas participantes do grupo de reabilitação pulmonar. Information Systems, 6, 20-43.

MacRae, W. D., \& Towers, G. H. (1984). Justicia pectoralis: a study of the basis for its use as a hallucinogenic snuff ingredient. Journal of ethnopharmacology, 12(1), 93-111. Ireland.

Malosh, R. E., Martin, E. T., Ortiz, J. R., \& Monto, A. S. (2018). The risk of lower respiratory tract infection following influenza virus infection: A systematic and narrative review. Vaccine, 36(1), 141-147.

Marcos, A., Bezerra, E., \& Tertuliano, F. (2006). Rendimento de biomassa, óleo essencial, teores de fósforo e potássio de chambá em resposta à adubação orgânica e mineral. Revista Ciência Agronômica, 37(2), 124-129.

McDade, L. A., Daniel, T. F., \& Kiel, C. A. (2008). Toward a comprehensive understanding of phylogenetic relationships among lineages of Acanthaceae s.1. (Lamiales). American journal of botany, 95(9), 1136-1152. United States.

Melo, C. R., Lira, A. B., Alves, M. F., \& Lima, C. M. B. L. (2017). O uso de plantas medicinais para doenças parasitárias. Acta Brasiliensis, 1(1), 28.

Mesquita, M. O. M. de, Pinto, T. M. F., \& Moreira, R. F. (2017). Potencial antimicrobiano de extratos e moléculas isolados de plantas da Caatinga: uma revisão. Fundação Oswaldo Cruz. Farmanguinhos. Núcleo de Gestão em Biodiversidade e ....

Moura, C. T. M., Batista-Lima, F. J., Brito, T. S., Silva, A. A. V, Ferreira, L. C., Roque, C. R., Aragão, K. S., et al. (2017). Inhibitory effects of a standardized 
extract of Justicia pectoralis in an experimental rat model of airway hyper-responsiveness. The Journal of pharmacy and pharmacology, 69(6), 722-732. England.

Mpiana, P. T., \& Kitadi, J. M. (2019). Justicia secunda Vahl species: Phytochemistry, Pharmacology and Future Directions : a mini-review. Discovery Phytomedicine, 6(4).

Oliveira, A. F. M., Xavier, H. S., Silva, N. H., \& Andrade, L. H. C. (2000). Screening cromatográfico de acanthaceae medicinais: Justicia pectoralis jacq. e J. gendarussa Burm. Revista Brasileira de Plantas Medicinais, 3(1), 37-41.

Pabón, L. C., Rodríguez, M. F., \& Hernández-Rodríguez, P. (2017). Plantas medicinales que se comercializan en Bogotá (Colombia) para el tratamiento de enfermedades infecciosas. Boletín Latinoamericano y del Caribe de Plantas Medicinales y Aromáticas, 16(6), 529-546. https://www.redalyc.org/pdf/856/85653615002.pdf\%0Awww.blacpma.usach.cl

Pérez Alonso, N., \& Jiménez, E. (2011). Producción de metabolitos secundarios de plantas mediante el cultivo in vitro. Biotecnología vegetal, 11(4), 195-211. https://biblat.unam.mx/pt/revista/biotecnologia-vegetal/articulo/produccion-de-metabolitos-secundarios-de-plantas-mediante-el-cultivo-in-vitro

Pietruszewska, W., Barańska, M., \& Wielgat, J. (2018). Place of phytotherapy in the treatment of acute infections of upper respiratory tract and upper gastrointestinal tract. Otolaryngologia polska $=$ The Polish otolaryngology, 72(4), 42-50. Poland.

Pitta, L. S. da C., Hipolito, R. L., Rocha, L. C. dos S., Sales, F. M., Silva, L. P. M. da, \& Flores, P. V. P. (2021). Alterações tomográficas pulmonares no COVID-19: revisão integrativa da literatura. Research, Society and Development, 10(13), e308101321194.

Prato, M. I. C., Silveira, A. da, Neves, E. T., \& Buboltz, F. L. (2014). Doenças respiratórias na infância: uma revisão integrativa, 14(1), 33-39. https://journal.sobep.org.br/article/doencas-respiratorias-na-infancia-uma-revisao-integrativa/

Profice, S. R., \& Andreata, R. H. P. (2011). Revisão taxonômica de Aphelandra R. Br. (Acanthaceae) de corola curto-bilabiada. Pesquisas. Botanica, 38(62), 7-70. Instituto Anchietano de Pesquisas. https://biblat.unam.mx/pt/revista/pesquisas-botanica/articulo/revisao-taxonomica-de-aphelandra-r-br-acanthaceae-decorola-curto-bilabiada

Prudente, R. C. C., \& Moura, R. B. de. (2013). Evidências Científicas Para a Indicação Popular De Algumas Espécies Da Família Rutaceae No Tratamento De Doenças Respiratórias Na Região Sudeste Do Brasil. Infarma - Ciências Farmacêuticas, 25(1), 24-31.

Ribeiro, L. H. L. (2019). Análise dos programas de plantas medicinais e fitoterápicos no Sistema Único de Saúde (SUS) sob a perspectiva territorial. Ciencia \& saude coletiva, 24(5), 1733-1742.

Rockenbach, A. P., Rizzardi, M. A., Nunes, A. L., Bianchi, M. A., Caverzan, A., \& Schneider, T. (2018). Interferência entre plantas daninhas e a cultura: alterações no metabolismo secundário. Revista Brasileira de Herbicidas, 1(1), 59.

Rodríguez Chanfrau, J. E., López Hernández, O. D., Núñez Figueredo, Y., Rodríguez Ferrada, C., \& Nogueira Mendoza, A. (2013). Obtention of dry extract from aqueous extracts of Justicia pectoralis Jacq. (tilo). Revista Cubana de Plantas Medicinales, 18(4), 543-554.

Rodríguez Chanfrau, J. E., \& Rodríguez Ferrada, C. (2014). Harvest time influences on coumarin and umbelliferone contents in extracts of Justicia pectoralis Jacq. (tilo). Revista Cubana de Farmacia, 48(3), 477-485.

Rodríguez Chanfrau, J., López Hernández, O., \& Gil Apan, J. (2008). Método para la cuantificación de cumarina en extracto seco a partir de extractos de Justicia pectoralis Jacq. Rev. cuba. plantas med, 13(3), $0-0$.

Rosina Maria Carvalho Caminha Muniz, Andre Luiz Cunha Cavalcante, Luis Mario Silva Pereira, Francisca Clea Florenco de Sousa, Silvania Maria Mendes de Vasconcellos, Danielle Silveira Macedo, Glauce Socorro de Barros Viana, M. M. de F. F. (2012). Plantas medicinais da renisus de atuação central. Infarma, 24(1-3), 74-81.

Ruzza, D. A. C., Göttert, V., Rossi, A. A. B., Dardengo, J. de F. E., \& Silva, I. V. da. (2014). Levantamento Etnobotânico no Município de Alta Floresta,Mato Grosso, Brasil. Enciclopédia Biosfera, 10(18), 3331-3343.

Saraiva, M. E., Ulisses, A. V. R. D. A., Ribeiro, D. A., Oliveira, L. G. S. De, Macêdo, D. G. De, Sousa, F. D. F. S. De, Menezes, I. R. A. De, et al. (2015). Plant species as a therapeutic resource in areas of the savanna in the state of Pernambuco, Northeast Brazil. Journal of Ethnopharmacology, 171, 141-153. Elsevier. Retrieved from http://dx.doi.org/10.1016/j.jep.2015.05.034

Sarmento Gadelha, C., Pinto Junior, V. M., Santos Bezerra, K. K., Borges Maracajá, P., \& Martins, D. S. S. (2015). Utilização de medicamentos fitoterápicos e plantas medicinais em diferentes segmentos da sociedade. Revista Verde de Agroecologia e Desenvolvimento Sustentável, 10(3), 1-15. https://www.gvaa.com.br/revista/index.php/RVADS/article/view/3564\%0Ahttp://fi-admin.bvsalud.org/document/view/r6uxu

Semenya, S. S., \& Maroyi, A. (2018). Data on medicinal plants used to treat respiratory infections and related symptoms in South Africa. Data in Brief, 21, 419-423. Elsevier Inc. http://dx.doi.org/10.1016/j.dib.2018.10.012

Da Silva, G. V. F. de C., Nogueira, L. T., Araujo, I. F. de B., De Souza, D. R., De Sousa, G. A., \& Da Silva, A. D. (2020). Tendências De Mortalidade Por Doenças Respiratórias E Doenças Cardiovasculares Em Teresina-Pi. SANARE - Revista de Políticas Públicas, 19(1), 7-17.

Troeger, C., Blacker, B., Khalil, I. A., Rao, P. C., Cao, J., Zimsen, S. R. M., Albertson, S. B., et al. (2018). Estimates of the global, regional, and national morbidity, mortality, and aetiologies of lower respiratory infections in 195 countries, 1990-2016: a systematic analysis for the Global Burden of Disease Study 2016. The Lancet Infectious Diseases, 18(11), 1191-1210.

Tseliou, M., Pirintsos, S. A., Lionis, C., Castanas, E., \& Sourvinos, G. (2019). Antiviral effect of an essential oil combination derived from three aromatic plants (Coridothymus capitatus (L.) Rchb. f., Origanum dictamnus L. and Salvia fruticosa Mill.) against viruses causing infections of the upper respiratory tract. Journal of Herbal Medicine, 17-18, 100288. Retrieved from https://www.sciencedirect.com/science/article/pii/S2210803319300351

Venâncio, E. T., Rocha, N. F. M., Rios, E. R. V, Feitosa, M. L., Linhares, M. I., Melo, F. H. C., Matias, M. S., et al. (2011). Anxiolytic-like effects of 
Research, Society and Development, v. 10, n. 16, e26101623152, 2021

(CC BY 4.0) | ISSN 2525-3409 | DOI: http://dx.doi.org/10.33448/rsd-v10i16.23152

standardized extract of Justicia pectoralis (SEJP) in mice: Involvement of GABA/benzodiazepine in receptor. Phytotherapy research : PTR, 25(3), 444-450. England.

Venegas-Casanova, E. A., Ruiz-Reyes, S. G., Gavidia-Valencia, J. G., Jara-Aguilar, R., Uribe-Villarreal, J. C., Curo-Vallejos, Y. F., Rengifo-Penadillos, R. A., et al. (2018). Variability in the chemical composition of Justicia pectoralis Jacq. (two varieties): essential oils in over several months. Pharmacology Online , 3, 402-411. Salerno: Italo-Latin American Society of Ethnomedicine (SILAE).

de Vries, J. X., Tauscher, B., \& Wurzel, G. (1988). Constituents of Justicia pectoralis Jacq. 2. Gas chromatography/mass spectrometry of simple coumarins, 3phenylpropionic acids and their hydroxy and methoxy derivatives. Biomedical \& environmental mass spectrometry, 15(8), 413-417. England.

Wasshausen, D. C., \& Wood, J. R. I. (2004). Acanthaceae of Bolivia. Contributions from the United States National Herbarium, 49, 1-152. Department of Botany, Smithsonian Institution. http://www.jstor.org/stable/23493155

World Health Organization. (2014). Infection prevention and control of epidemic-and pandemic prone acute respiratory infections in health care. WHO Library.

Wu, W.-F., Wang, J.-N., Li, Z., Wei, B., Jin, J., Gao, L., Li, H.-D., et al. (2020). 7-Hydroxycoumarin protects against cisplatin-induced acute kidney injury by inhibiting necroptosis and promoting Sox9-mediated tubular epithelial cell proliferation. Phytomedicine: international journal of phytotherapy and phytopharmacology, 69, 153202. Germany.

Yordan Martínez Aguilar, C., Rodríguez, F. S., Manuel Almeida Saavedra, C., Espinosa, R. H., \& Yero, O. M. (2012). Secondary metabolites and in vitro antibacterial activity of extracts from Anacardium occidentale L. (Cashew tree) leaves. Revista Cubana de Plantas Medicinales, 17(4), 320-329.

Zumla, A., \& Niederman, M. S. (2015, May). Respiratory tract infections in community and healthcare settings: epidemiological, diagnostic and management challenges. Current opinion in pulmonary medicine. United States. 